\author{
Nadine Honke ${ }^{\star, 1,4}$, Namir Shaabani ${ }^{*, 1,2,4}$, Dong-Er Zhang ${ }^{3}$, Cornelia Hardt ${ }^{1}$ and Karl S Lang ${ }^{1}$
}

Since the discovery of the ubiquitin system and the description of its important role in the degradation of proteins, many studies have shown the importance of ubiquitin-specific peptidases (USPs). One special member of this family is the USP18 protein (formerly UBP43). In the past two decades, several functions of USP18 have been discovered: this protein is not only an isopeptidase but also a potent inhibitor of interferon signaling. Therefore, USP18 functions as 'a' maestro of many biological pathways in various cell types. This review outlines multiple functions of USP18 in the regulation of various immunological processes, including pathogen control, cancer development, and autoimmune diseases.

Cell Death and Disease (2016) 7, e2444; doi:10.1038/cddis.2016.326; published online 3 November 2016

Facts

- There are two functional domains of USP18, one is responsible for isopeptidase activity and the other one inhibits type I interferon signaling by blocking the type I interferon receptor 2 (IFNAR2) subunit.

- USP18 is involved in the development of $\mathrm{CD} 11 \mathrm{~b}^{+}$dendritic cells (DCs) and Th17 cells.

- The anti-IFN-I effect of USP18 leads to enforced viral replication, which helps to activate the adaptive immune system. In case of an infection with a virus that resembles autoantigen, enforced viral replication may lead to autoimmune diseases.

- USP18 has an important role in tumorigenesis.

\section{Open questions}

- Development of potential therapeutic molecules to target USP18.

- Characterize in more detail the role of both isoforms of USP18 in humans and determine whether the regulation pathway can influence the outcome of its function.

- Study the role of USP18 during vaccination.

\section{Structure and functional domains of USP18}

Ubiquitin-specific peptidase 18 (USP18) is known as an ISG15 isopeptidase and a negative regulator of type I and type III interferon signaling. ${ }^{1,2}$ The Usp18gene was originally called Ubp43 because it encodes a 43-kDa protein homologous with ubiquitin-specific proteases (UBPs). It was first cloned by Liu et all $^{3,4}$ from mice expressing the leukemia fusion protein AML1-ETO and later by other groups from virus-infected porcine alveolar macrophages ${ }^{5}$ and human melanoma cell lines. ${ }^{6}$ The gene and protein were renamed Usp18 and USP18, respectively, according to the systematic nomenclature suggested by Baker et al. ${ }^{7}$ for UBPs and ubiquitin-like (Ubl) proteins. On the basis of its amino-acid sequence, USP18 is a member of the USP family, which is responsible for removing ubiquitin or $\mathrm{Ubl}$ proteins from their conjugated substrates.

The Usp18 gene spans $25.01 \mathrm{~kb}$ on mouse chromosome 6 and includes 11 exons. The transcript of $1771 \mathrm{bps}$ is translated as a protein with 368 amino acids (aa). The protein shares catalytic domains of UBPs. In the mouse, a mutation of the USP 18 protein within the Cys box at position 61 completely abolishes the isopeptidase activity of the protein by replacing the active site of cysteine $\mathrm{C} 61$ with codon specific for alanine C61A. However, not only the Cys box is responsible for isopeptidase function, but also the His box and the Asn

\footnotetext{
${ }^{1}$ Institute of Immunology, Medical Faculty, University of Duisburg-Essen, Hufelandstr. 55, Essen 45147, Germany; ${ }^{2}$ Department of Immunology and Microbial Science, The Scripps Research Institute, La Jolla, CA 92037, USA and ${ }^{3}$ Moores UCSD Cancer Center, University of California San Diego, La Jolla, CA 92093, USA ${ }^{*}$ Corresponding author: N Honke or N Shaabani, Institute of Immunology, Medical Faculty, University of Duisburg-Essen, Hufelandstr. 55, Essen 45147, Germany. Tel: +49 201723 4247; Fax: +49 201723 5410; E-mail: nadine_honke@yahoo.de or namirshaabani@ hotmail.com

${ }^{4}$ These authors contributed equally to this work.

Abbreviations: Aa, amino acids; APL, acute promyelocytic leukemia; Asn, asparagine; BMDC, bone marrow dendritic cell; $C \mathrm{CL}$, chemokine (C-C motif) ligand; CML, chronic myelogenous leukemia; CNS, central nervous system; CTL, cytotoxic T lymphocyte; CXCL, chemokine (C-X-C motif) ligand; Cys, cysteine; DC, dendritic cell; DGCR, DiGeorge syndrome critical region gene; EAE, experimental autoimmune encephalomyelitis; EGFR, epidermal growth factor receptor; ENU, $N$-ethyl- $N$-nitrosourea; FASL, Fas ligand; HBV, hepatitis B virus; HCV, hepatitis C virus; His, histidine; HIV, human immunodeficiency virus; IFN-I, type I interferon; IFNAR, type I interferon receptor; IFNLR, type III interferon receptor; IL, interleukin; IP, interferon gamma-induced protein; ISG, interferon-stimulated gene; JAK, janus-activated kinase; LCMV, lymphocytic choriomeningitis virus; LPS, lipopolysaccharide; MIBC, muscle-invasive bladder cancer; MS, multiple sclerosis; NEMO, NF- $\kappa B$ essential modulator; PEPT, peptide transporter; Poly I:C, polyinosinic-polycytidylic acid; PRRSV, porcine reproductive and respiratory syndrome virus; PyVmT, polyomavirus middle T; RANKL, receptor activator of NF-kB ligand; siRNA, small-interfering ribonucleic acid; SNP, single-nucleotide polymorphism; SNV, sindbis virus; STAT, signal transducer and activator of transcription; TAB, TAK1-binding protein; TAK, transforming growth factor beta-activated kinase; Th, Thelper; TLR, toll-like receptor; TNF- $\alpha$, tumor necrosis factor alpha; TNFR, tumor necrosis factor receptor; TRAIL, (TNF)-related apoptosis-inducing ligand; TYK, tyrosine kinase; Ubl, ubiquitin-like; UBP, ubiquitin-specific protease; USP, ubiquitin-specific peptidase; VSV, vesicular stomatitis virus; WT, wild-type; WT1, wilms tumor protein 1

Received 20.6.16; revised 12.9.16; accepted 16.9.16; Edited by T Brunner
} 


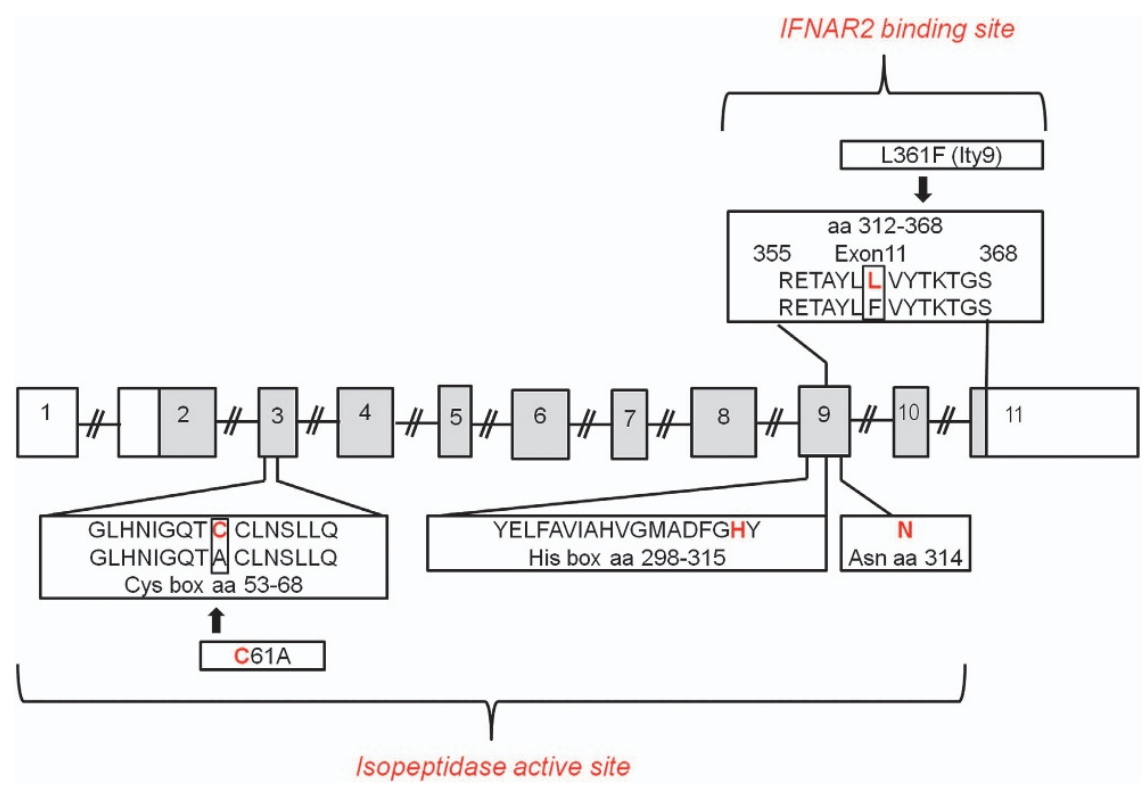

Figure 1 Structure and functional domains of Usp18. The figure shows the structure of Usp18 in Mus musculus. Usp18 is localized on chromosome 6 and consists of 11 exons. The protein is characterized by two functional domains: The isopetidase activity site and IFNAR-binding site. For the isopeptidase function, two boxes (Cys, His) and the amino acid Asn at position 314 are essential. A mutation in $\mathrm{C}, \mathrm{H}$, or N (red), respectively, can abolish the isopeptidase activity. The IFNAR2 binding site is located from exons 9-11. A mutation in amino-acid position 361 from Leucine (L, red) to Phenylalanine $(F)$ in exon 11 disables USP18 protein from binding to the IFNAR2 subunit

residue $^{8}$ (Figure 1). Another functional domain specific for USP18 has been mapped from exon 9-11 (aa 312-368); this domain facilitates binding to the intracellular domain of the IFNAR2 subunit in order to regulate interferon signaling ${ }^{9}$ (Figure 1). Binding to IFNAR2 is abolished by a mutation at position 361 of the USP18 protein, ${ }^{10}$ a mutation that was induced by $\mathrm{N}$-ethyl- $\mathrm{N}$-nitrosourea mutagenesis, ${ }^{11}$ and mouse strains carrying this mutation are called Usp $18^{l t y}$.

In humans, two isoforms of USP18 have been described. ${ }^{12}$ They differ in their $\mathrm{N}$-terminal region because of translation from a non-canonical rare start codon CUG; this translation produces a full-length protein or a canonical start codon, AUG, and results in the $N$-terminal truncated isoform USP18-sf. Although USP18 is mainly located in the cytoplasm, USP18-sf is evenly distributed in the cytoplasm and the nucleus. Both proteins maintain their functional activity in term of enzymatic and IFNAR2 binding ability, but the USP18-sf isoform is the main delSGylation enzyme for nuclear proteins and therefore may serve cell-specific functions. In addition, because USP18-sf is controlled by two independent mechanisms, its regulation is more flexible. Additional studies are needed to characterize the role of each isoform and to determine whether the regulation pathway can influence the outcome of USP18 function. $^{12}$

\section{Expression of USP18}

USP18 expression is detectable in several tissues but at different levels. For instance, high expression of Usp18 has been measured in liver, spleen, and thymus. ${ }^{3,13}$ However, a low but clearly detectable level of USP18 expression has been seen in bone marrow, adipose tissue, and lung tissue. ${ }^{3}$
The expression of USP18 has been studied in a wide spectrum of cells. For example, in our earlier studies we detected high levels of USP18 in CD169 ${ }^{+}$macrophages and bone marrow-derived DCs, but we found no USP18 in lung fibroblasts and bone marrow-derived macrophages. ${ }^{14,15} \mathrm{~A}$ high level of USP18 expression has also been measured in peritoneal macrophages and monocyte-derived macrophages $^{3,13}$ and in two murine monocyte-related cell lines, RAW 264.7 and M1. ${ }^{3}$ USP18 is also expressed in various lymphatic and hematopoietic cell populations, including splenic Tand B cells. In T cells, USP18 is highly expressed in naive, effector/memory, and natural regulatory $\mathrm{T}$ cells. ${ }^{16}$ Microarray data indicate that a high level of Usp18 expression is also maintained in T helper 0 , Th1, and Th17 cells but is diminished in Th2 cells and inducible regulatory $\mathrm{T}$ cells. ${ }^{16}$ However, the expression level of USP18 is differently regulated during $T$ cell activation, tolerance, and effector differentiation. ${ }^{16}$ USP18 is induced by IFN- $\beta$ not only in lymphocytes but also in $\mathrm{HO}-1$ human melanoma cells, ${ }^{6}$ in Huh-7.5 cells treated with irrelevant small-interfering RNAs, ${ }^{17}$ in the choroid plexus and in ependymal cells. ${ }^{18}$ Moreover, transfecting human hepatoblastoma HepG2 cells with hepatitis $B$ virus (HBV) genome increased the expression of USP18 in those cells. ${ }^{19}$

The Usp18 gene is rapidly and strongly upregulated after viral infection or by type I and type III IFNs, ${ }^{1,6,13,19,20}$ lipopolysaccharide (LPS), ${ }^{21,22}$ tumor necrosis factor alpha $(\mathrm{TNF}-a){ }^{22}$ or genotoxic stress ${ }^{13,23}$ (Figure 2a). USP18 is degraded by proteasomes. ${ }^{24,25}$ In most species, USP18 maps to a different chromosome (Table 1).

The absence of USP18 strengthens the signaling of IFN-I and IFN-III;, ${ }^{1,2}$ and is associated with prolonged Janusactivated kinase/signal transducer and activator of 


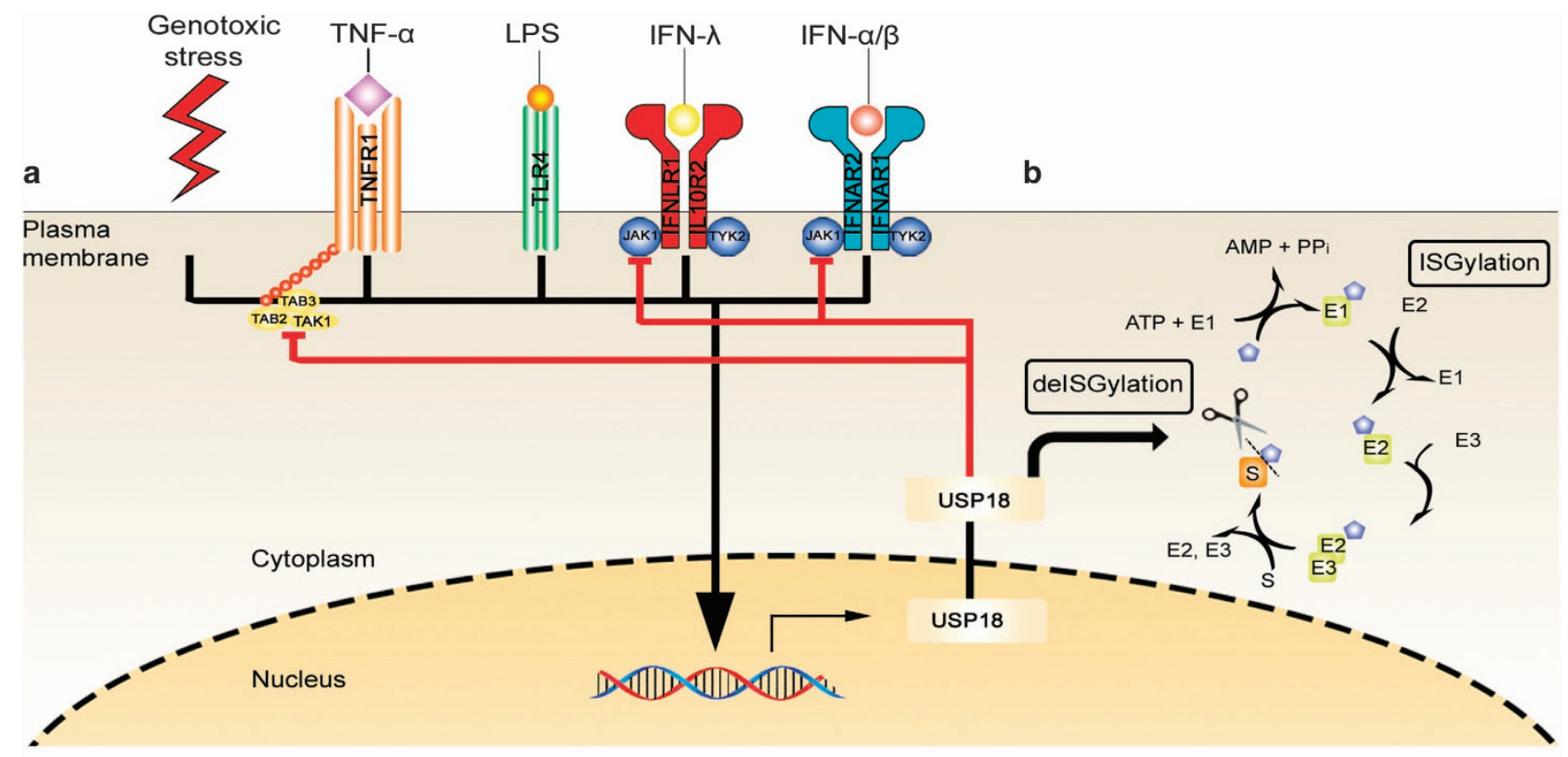

E1 Enzyme 1 (UBE1L)

E2 Enzyme $2(\mathrm{UBCH} 8)$

E3 Enzyme 3 (HERC5, EFP, HHARI)
S Substrate

- ISG15

- Inhibition

Figure 2 Regulation and function of USP18. (a) USP18 is upregulated through different signaling pathways (genotoxic stress, TNFR1, TLR4, IFNLR1//L1OR2, and IFNAR1/ IFNAR2). USP18 itself regulates signaling through a negative feedback mechanism either by inhibiting the binding of JAK1 to the IFNAR2 subunit or the IFNLR1 subunit, or by inhibiting the ubiquitination of the TAK1-TAB complex of TNFR1. (b) After ISGylation of ISG15 via a three enzymatic process, USP18 can deISGylate the ubiquitin-like protein ISG15 from ISG15-conjugated substrate by cleaving the isopeptide bond between ISG15 and the substrate. Lacking of USP18 leads to an increase signaling of IFN-I, IFN-III, TNF- $\alpha$ and high levels of conjugated ISG15

Table 1 Chromosomal location of Usp18 in various species

\begin{tabular}{lc}
\hline Species & Chromosome \\
\hline Danio reri (Zebrafish) & 4 \\
Xenopus (Silurana) tropicalis (Western clawed frog) & Unknown \\
Sus scrofa (Pig) & 5 \\
Canis lupus familiaris (Dog) & 27 \\
Macaca mulatta (Rhesus monkey) & 10 \\
Bos taurus (Cattle) & 5 \\
Rattus norvegicus (Norway rat) & 4 \\
Mus musculus (Mouse) & 6 \\
Homo sapiens (Human) & 22 \\
\hline
\end{tabular}

transcription (JAK-STAT) signaling. In addition, absence of USP18, which cleaves ISG15 from its target protein, prolongs ISG15-mediated ISGylation. ${ }^{2,9}$ Usp18-deficient cells exhibit high sensitivity to treatment with IFN-I, Poly I:C, and LPS. Treating Usp18-deficient hematopoietic cells with Poly I:C decreases the number of white blood cells since apoptosis is not prevented by USP $18 .{ }^{26}$ Moreover, knocking down USP18 markedly enhances the NF- $\kappa \mathrm{B}$ signaling induced by various TLR ligands. ${ }^{23}$ A study using an oncogenic cell line ( $E 1 A$ cells) found that USP18 activates the extrinsic TNF-related apoptosis-inducing ligand (TRAIL) pathway after IFN- $a$ challenge. ${ }^{27}$
In human promonocytic THP-1 cells, the expression of proinflammatory cytokines such as TNF- $a$, interleukin-6 (IL-6), and IL-1 $\beta$ is significantly higher when USP18 is silenced with siRNA. ${ }^{23}$ Interestingly, in contrast to E1A cells, Usp18deficient murine bone marrow cells and THP-1 cells that have been treated with IFNa/ $\beta$ do not experience apoptosis after treatment with TRAIL or FASL. ${ }^{28}$ However, IFN- $\alpha / \beta$ still triggers apoptosis in these cells through the mitochondrial pathway and the reactive oxygen species pathway, ${ }^{28}$ a finding indicating that USP18 influences cell survival in various pathways depending on the cell type.

\section{Usp18-deficient mice}

The generation of Usp18-deficient mice gave researchers the opportunity to examine many immunological mechanisms and disorders. ${ }^{18}$ Initially, Usp $18^{-1-}$ mice were generated on a mixed Sv129-C57BL/6 background. These mice are born at a normal ratio of WT to heterozygous littermates; however, homozygous knockout mice die of hydrocephalus 2-19 weeks after birth. ${ }^{18}$ Further analysis showed that the difficulties in generating viable Usp $18^{-/-}$mice on a C57BL/6 background occurred because the deletion of USP18 results in an increase in the concentration of free and conjugated ISG15 at the fetomaternal interface and leads to fetal death. ${ }^{29}$ 
Interestingly, knockout mice generated on the FVB/N genetic background are more viable and do not exhibit the severe neurological symptoms that occur in mice generated on a C57BL/6 background, which develop brain injury because of necrosis of ependymal cells. ${ }^{18}$ This finding indicates that strain-specific modifiers may influence the neurological disorders and lethality induced by the absence of USP18. Although the role of ISG15 in embryonic development has been demonstrated, ${ }^{29}$ the neurological disorders in Usp $18^{-/}$ mice are not related to high expression of ISG15, because hydrocephalus also develops in mice with double knockout of Usp18 and Isg15. ${ }^{30}$ Homozygous Usp18-deficient mice are hypersensitive to IFN-I and die within $72 \mathrm{~h}$ after treatment with synthetic double-stranded RNA Poly I:C. ${ }^{26}$ A recent study showed that strong signaling of IFN-I in Usp $18^{-/}$mice increases RANKL-mediated osteoclast differentiation and elevates the induction of osteoclastogenic cytokines such as IP-10 and IL-6, which causes osteopenia. ${ }^{31}$

Usp18 knockout mice are more resistant to intracerebral infection with LCMV and VSV ${ }^{32}$ but also display increased mortality to intravenous viral infection, as discussed below.

To study specifically the enzymatic activity of USP18, Ketscher et al. generated a mutant mouse strain that lacks the isopeptidase activity of USP18. Using a knock-in targeting strategy, they replaced the enzymatic active site of cysteine (C61) with a codon specific for alanine (C61A). In contrast to Usp18 knockout mice, Usp18 ${ }^{\mathrm{C} 61 \mathrm{~A} / \mathrm{C6} 1 \mathrm{~A}}$ mice do not exhibit brain abnormalities or increased lethality. They exhibit high levels of ISG15 conjugates but normal IFN-I signaling. ${ }^{33}$

\section{Functions of USP18}

\section{Role of Usp18 in cell development}

$C D 11 b^{+}$DCs: USP18 is involved in the development of conventional CD11 $\mathrm{b}^{+} \mathrm{DCs}$. An in vitro study showed that the generation of BMDCs by Usp18-deficient mice is impaired. This defect is due to the suppressive role of Usp18 on the IFN-I pathway, because DC development is also impaired after treatment with IFN-I. In vivo, the spleen and bone marrow of Usp18-deficient mice contain fewer CD11 ${ }^{+}$DCs than WT mice. Conventional CD11 $b^{+}$DCs are developed via the IFN-I pathway independent of the role of USP18 in deconjugation of the Ubl protein ISG15. ${ }^{34}$

TH17 cells: During infection, $\mathrm{CD}^{+} \mathrm{T}$ cells have an important role. $\mathrm{CD}^{+} \mathrm{T}$ cells differentiate into separate subtypes that produce distinct effector cytokines. The main subtypes are Th1 cells (which produce IFN- $\gamma$ ), Th2 cells (IL-10), and Th17 cells (IL-17, IL-17F, IL-21, and IL-22). ${ }^{35}$ Th17 cells are involved in autoimmune diseases such as multiple sclerosis (MS). Recently, Liu et al. ${ }^{16}$ found that USP18 is necessary for Th17 differentiation and autoimmune response. USP18 can inhibit the ubiquitination of the TAK1-TAB complex, thereby inhibiting IL-2 production and promoting IL-17 production and synthesis.

\section{Role of USP18 as an ubiquitin-specific peptidase}

In mammalian cells, many proteins are modified by ubiquitination, a process which is important for different vital events. For instance, the ubiquitin pathway is essential for cell cycle, ${ }^{36}$ cell differentiation, and proliferation. ${ }^{37}$ This pathway mediates protein degradation, ${ }^{38}$ turnover of transport proteins, ${ }^{39}$ and transcription activation. ${ }^{40}$ At the immune level, this pathway is essential for antigen presentation. ${ }^{41}$ These mechanisms are controlled by a wide spectrum of enzyme family members called UBPs, which remove ubiquitin from a large range of protein substrates. ${ }^{42}$ The family members vary in size and in amino acid sequences, but they all share six conserved regions. ${ }^{8}$ UBP43 (Usp18) is one member of this ubiquitin protease family; ${ }^{3-5,13,42,43}$ it controls the protein binding of $\mathrm{Ubl}$ protein ISG15. After stimulation with IFN- $\alpha / \beta$, ISG15 is upregulated and conjugated to various cellular substrates through a three-step enzymatic cascade: ${ }^{44,45}$ E1-activating enzyme (UBE1L), ${ }^{46}$ E2-conjugating enzyme (UBCH8), ${ }^{47,48}$ and various E3 ligases ${ }^{49,50}$ (Figure 2b). This conjugation mechanism is reversible and is controlled by the isopeptidase active site of IFN-inducible cysteine protease USP18. ${ }^{13}$ This functional domain includes the cysteine box with a cysteine residue, a histidine box with a histidine residue as well as an adjacent asparagine. These sequences are necessary for the function of the enzymatic activity of USP18. ${ }^{42,51}$

Cross-talk occurs between ISG15 and USP18. ISG15 itself can stabilize USP18; this stabilization is important for preventing the undesirable autoinflammatory effect of sustained IFN- $\alpha / \beta .^{52}$ Interestingly, this phenotype was observed only in human cells but not in murine cells. ${ }^{53}$ Unlike humans, IFN-I signaling is downregulated in mice regardless of ISG15 competency. ${ }^{53}$ In addition to ISG15, USP18 also specifically inhibits K63-linked ubiquitination of NEMO, leading to the negative regulation of NF- $\kappa B$ activation induced by the TAK1TAB complex. ${ }^{16,23}$ A recent study found that USP18 can enhance the cellular transport rate in Xenopus laevis oocytes by increasing the activity of PEPT1 and PEPT2; this activation was believed to be due to the reversal of ubiquitination and the subsequent degradation of carrier protein. ${ }^{54}$

\section{Role of USP18 during viral infection}

Interferon-I signaling is mastered through the binding of IFN-I to the heterodimeric interferon receptor, which consists of two subunits: IFNAR1 and IFNAR2. Both subunits are associated with kinase activity. IFN-I binding leads to phosphorylation of JAK1 and TYK2 and in turn to phosphorylation of STATs, which initiate the transcription of antiviral genes by binding to the interferon-stimulated response element. ${ }^{55}$ USP18 is considered to be a negative regulator of the IFN-I pathway by inhibiting JAK-STAT signaling ${ }^{13,26}$ (Figure 2a). This function is independent of its activity as an isopeptidase. ${ }^{9}$ USP18 itself is upregulated in the presence of IFNs through the activation of the JAK-STAT signaling pathway. ${ }^{6,20}$ In turn, USP18 specifically binds to the IFNAR2 subunit and thereby prevents the phosphorylation of JAK1 by blocking the interaction of JAK1 and the IFNAR2 subunit and downstream signaling. ${ }^{9}$ This phenotype cannot be explained by the delSGylation activity of USP18, because deleting ISG15 or the ISGylation-activating 
enzyme UBE1L in mice did not reverse the phenotype in Usp18-deficient mice. ${ }^{30,56,57}$ However, unlike mice, the presence of ISG15 in human cells is essential to stabilize USP18 and guarantee the function of this protein as IFN-I suppressor. ${ }^{53}$ This means that, in humans, ISG15 can also be considered as a good target protein by which we can strengthen IFN-I signaling and reduce viral replication.

In the absence of USP18, the phosphorylation of STAT1 and STAT2 is prolonged, ${ }^{58}$ and the expression of hundreds of antiviral genes, chemokines, cytokines, and antigenpresenting genes is enhanced, as confirmed by microarray data. ${ }^{59}$ Additional in vitro studies found that the growth of PRRSV is restricted in Usp18-overexpressed MARC-145 cells. ${ }^{51,60}$ This restriction is due to increased nuclear translocation of transcription factor p65 and decreased nuclear translocation of $\mathrm{p50} .^{60}$ An in vivo study found that Usp18-deficient mice exhibit less replication of various viruses, including LCMV ${ }^{15}$ and VSV. ${ }^{14}$ The antiviral effect is not due to enhanced ISGylation of ISG15 in the absence of USP18, because Isg15-deficient mice do not exhibit any antiviral impairment after infection with LCMV or VSV. ${ }^{56}$ In Addition, Usp18 knockout mice exhibit less replication of several other viruses, such as $\mathrm{HBV},{ }^{61,62}$ Sindbis virus, ${ }^{63}$ influenza B virus, ${ }^{46} \mathrm{HIV}^{64}$ and others in which the ISGylation pathway is involved ${ }^{65-69}$ (Table 2).

Not only viral infections but also sterile inflammation, which can be induced through different stimuli (chemical, physical, or metabolic noxious), ${ }^{70}$ can increase the USP18 expression through certain inflammatory cytokines (TNF- $a$ and LPS), which impairs IFN-I responses and viral control. For example, ischemic reperfusion injury upregulates USP18 expression in the liver and induces an IFN- $a$ refractory state. ${ }^{22}$ That may raise the risk of an opportunistic viral infection.

Role of USP18 in enforced viral replication. The innate immune system reduces viral replication via IFN-I; this reduction is essential for inhibiting the spread of virus to other organs. Simultaneously, it reduces the presentation of viral antigens to cells of the adaptive immune response. Because the amount of antigen is positively correlated with the degree of activation of the adaptive immune system, ${ }^{71,72}$ IFN-I can act as a double-edged sword. In our previous work using mice infected with VSV, we found that macrophages capture virus particles and suppress viral replication in the liver and the red pulp of the spleen in an interferon-dependent manner. ${ }^{14}$ However, the high expression of the gene encoding the inhibitory protein USP18 in CD169 ${ }^{+}$metallophilic macrophages reduces the responsiveness to IFN-I, which allows locally restricted replication of virus. This replication is essential to activate the adaptive immune system and to prevent the fatal outcome of infection (Figure 3a). An additional study showed that the same mechanism can lead to autoimmune diabetes as discussed below.

\section{Role of USP18 in antibacterial response}

As shown above, USP18 influences numerous cellular signaling and biological functions. Consequently, many researchers have studied the role of USP18 during bacterial
Table 2 USP18 pathways that influence the replication of viruses

\begin{tabular}{lccc}
\hline Virus & ISGylation & JAK/STAT & References \\
\hline LCMV & & + & 56 \\
VSV & & + & 56 \\
Sindbis virus & + & 32,63 \\
HBV & + & 61,62 \\
HCV & + & $67-69$ \\
HIV & + & 64 \\
Influenza B virus & + & 46 \\
Coronavirus & + & 66 \\
Herpes virus & + & 65 \\
\hline
\end{tabular}

infection. The results show that strong IFN-I signaling resulting from the absence of USP18 leads to less growth of Salmonella typhimurium in Usp18 knockout mice than in control mice. ${ }^{21}$ However, survival rates are slightly higher for Usp18-deficient mice because of their hypersusceptibility to LPS challenge. A more recent study by Richer et al. found that a missense mutation (ENU-induced mutation) in Usp $18^{\text {lty } 9}$ mice leads to lethal susceptibility to $S$. typhimurium and that the bacterial titer in liver and spleen is higher in these mice than in WT mice. ${ }^{11}$ Moreover, infection with $S$. typhimurium enhances IFN-I signaling and inflammatory response in Usp18 ${ }^{\text {lty } 9}$ mice. This immunological response is explained by the impairment of STAT4 phosphorylation and IFN- $p$ production as the result of STAT1 hyperactivation. ${ }^{11}$ Another group of researchers attributed the susceptibility of Usp $18^{\text {ltyg }}$ mice to S. typhimurium or Mycobacterium tuberculosis infection to elevated levels of IL-10, IL-1 $\beta$, or IL-17, in addition to the deregulation of autophagy markers. ${ }^{10}$ Surprisingly, the contradictory nature of these two results obtained with Usp18 knockout mice and Usp $18^{\text {tty9 }}$ mice can be explained by either the different backgrounds of the mice or the specific ENU-induced mutation of Usp18. Other studies demonstrated the upregulation of USP18 during infection with Rickettsia conorii. As expected, this upregulation inhibits IFN-I-induced genes in human microvascular endothelial cells; however, whether USP18 plays a role in controlling bacterial infection has not yet been fully clarified. ${ }^{73,74}$

\section{Role of USP18 in autoimmune diseases}

Autoimmune diabetes. Autoimmune diabetes, also called diabetes mellitus type 1 , is characterized by the destruction of insulin-producing beta islet cells in the pancreas. The priming of autoreactive $\mathrm{CD}^{+} \mathrm{T}$ cells requires the presentation of autoantigen to $\mathrm{CD}^{+} \mathrm{T}$ cells. This presentation can occur during a viral infection that resembles cross-reactive epitopes of beta islet cells. DCs are considered to be mainly responsible for this process. A mouse model showed that the absence of DCs inhibits the viral replication that resembles autoantigen and, consequently, inhibits the onset of diabetes. Because DCs express more USP18 than do other types of cells, the lack of a response to the antiviral effect of IFN-I leads to augmented viral replication and a sufficient amount of autoantigen. Knocking down USP18 expression inhibits viral replication and consequently does not lead to autoimmune diabetes ${ }^{15}$ (Figure $3 b$ ). 
a

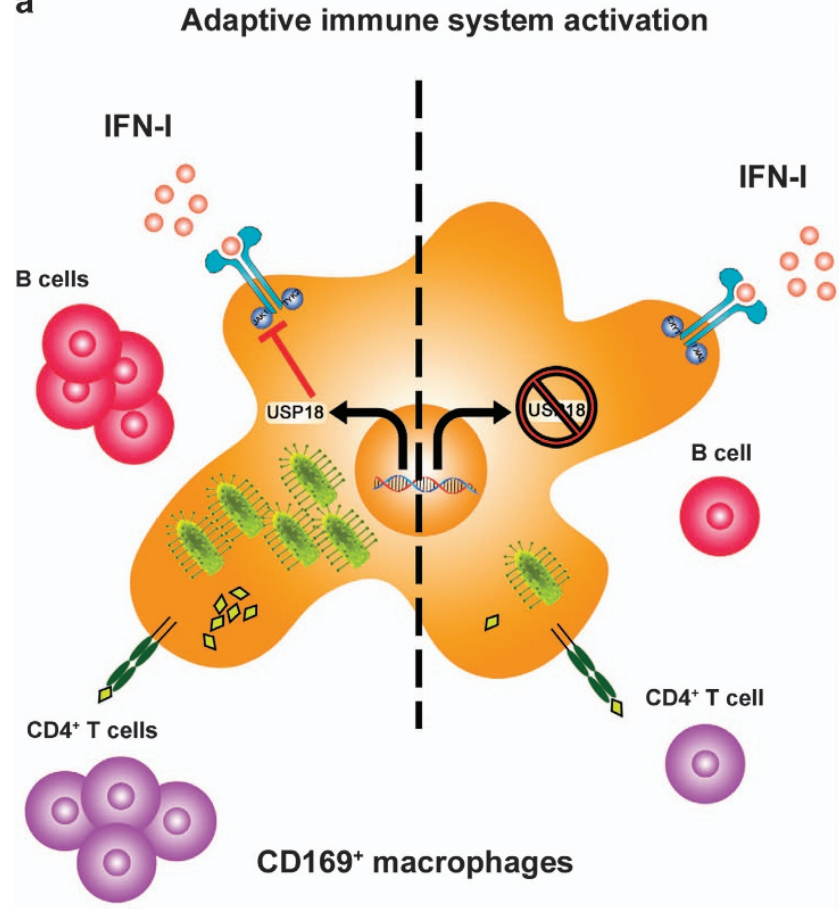

b

\section{Diabetes mellitus type I}

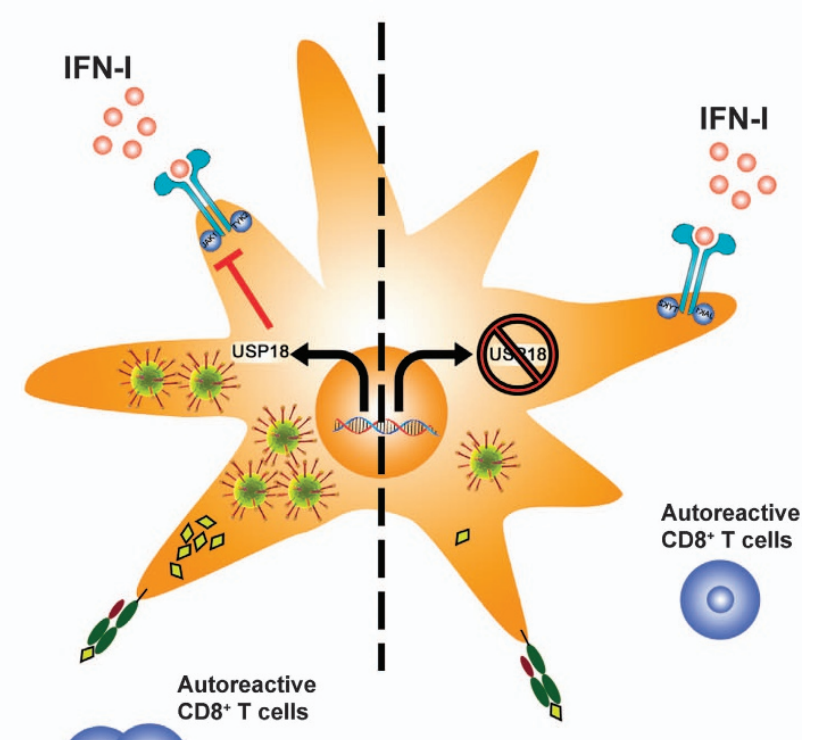

Dendritic cells

MHC-II complex

Virus

Virus carrying autoantigen

- Type I interferon (IFN-I)

MHC-I complex

Processed antigen

Figure 3 Role of USP18-dependent enforced viral replication in activation of the adaptive immune system and onset of diabetes mellitus type I. (a) USP18 inhibits IFNAR signaling, which leads to enforced viral replication in $\mathrm{CD}_{169^{+}}$macrophages. This replication guarantees sufficient amount of antigen, which is processed and presented via $\mathrm{MHC}$ complexes to activate the adaptive immune system. Lack of USP18 strengthens the IFN-I signaling, inhibits viral replication and reduces the presentation of processed antigen and consequently diminished adaptive immune system activation. (b) In case of enforced viral replication in dendritic cells of a virus resembling an autoantigen, autoreactive CD ${ }^{+}$ T cells will be primed, which leads to autoimmune diseases such as type I diabetes, whereas USP18 deficiency reduces the priming of autoreactive CD8 ${ }^{+} \mathrm{T}$ cells and onset of autoimmune diabetes owing to inhibition of enforced viral replication

Further studies show that the expression of USP18 by beta islet cells themselves is important for inhibiting diabetes. ${ }^{75,76}$ On the one hand, the upregulation of USP18 expression by IFN-I in beta islet cells prevents the activity of proinflammatory chemokines such as CCL5, CXCL10, and IL-15 and consequently inhibits insulitis. On the other hand, the upregulation of USP18 inhibits beta cell apoptosis. ${ }^{75,76}$

MS. IFN- $\beta$ is considered the first line of therapy against MS. ${ }^{77,78}$ IFN- $\beta$ can induce USP18 expression through IFNAR. $^{6}$ In turn, USP18 inhibits IFN- $\beta$ signaling. It was recently shown that USP18 is found in microglia, tissueresident macrophages in the central nervous system. These macrophages have an essential role in controlling tissue homeostasis, and disorders in the function of microglia can lead to neuroinflammatory diseases. The presence of USP18 in microglia is essential for keeping them in a quiescent state. ${ }^{79,80}$ When USP18 is absent, the microglia undergoes a prolonged period of activation of STATs, leading to microgliopathy. ${ }^{79}$

USP18 is also involved in the differentiation of $\mathrm{CD} 4^{+} \mathrm{T}$ cells into Th17 cells, as discussed above. Th17 cells are widely 
recognized as essential player for the development of MS. A study using Usp18-deficient mice found that the disease score of EAE is significantly lower than in WT mice, ${ }^{16}$ a finding that demonstrates the important role of USP18 in the progression of MS.

Interestingly, in humans, USP18 expression is lower in MS patients than in healthy persons. ${ }^{81}$ A genetic study found that MS is associated with two SNPs, rs9618216 (C/T) is located in the promoter region of USP18 and rs2542109 (A/G) in the fourth intron of the USP18 gene. The USP18 haplotype, TG is associated with risk to MS, whereas the haplotype $C G$ is protective. $^{82}$

\section{Impact of USP18 in tumor development}

Yan et al. $^{83}$ found that USP18 affects tumor progression because of its effects on interferon signaling. Using a wellestablished mouse model of BCR-ABL-induced chronic myelogenous leukemia-like myeloproliferative disease, they showed that USP18 has an important role in regulating the latency and severity of leukemia development. ${ }^{83}$ The absence of USP18 in the breast cancer cell line MCF-7 results in an increase in the induction of apoptosis by chemotherapy and treatment with IFN- $a{ }^{27}$ Silencing USP18 in glioblastoma cells produces similar results, ${ }^{84}$ a finding suggesting that strengthening the IFN-I pathway by silencing USP18 elicits apoptosis in drug-treated cells with robust caspase-8 and caspase- 3 activation independent of the mitochondrial pathway. ${ }^{27}$

The direct effect of USP18 on tumor progression has been studied with leukocytes. UBP43 can be considered as an antineoplastic target for the treatment of APL. Because USP18 directly regulates the growth of APL cells, knocking down USP18 reduces cell growth and induces apoptosis. ${ }^{85}$ In addition, USP18 expression has been detected in several types of human malignant tissues, such as kidney and prostate tissues. ${ }^{86,87} \mathrm{An}$ increase in USP18 expression specifically stabilizes cyclin D1, not only in a mouse model but also in clinical studies. ${ }^{86,88}$ Moreover, the role of UBE1L in repressing cyclin D1 has been described in diverse types of cancers. ${ }^{89,90}$ USP18 is also directly involved in cell proliferation: higher expression of USP18 is associated with faster proliferation. In both murine and human cell lines, an increase expression of USP18 is associated with the activity of WT1. WT1 binds directly to the Usp18 promoter and suppresses its transcription. These findings illustrate the important role of USP18 in tumorigenesis of the kidney. ${ }^{87}$

Another mechanism that rules out the importance of USP18 in tumor progression was found in a PyVmT model of mammary tumorigenesis. The absence of USP18 leads to the high production of $\mathrm{T}$ cell chemoattractant CXCL10 by mammary epithelial cells, and this increased production creates a tumor-suppressive microenvironment by recruiting $\mathrm{CD}^{+}{ }^{\mathrm{T}}$ cells. $^{2}$ In addition, USP18 has been found to inhibit TRAIL-induced apoptosis independently of the delSGylation pathway. ${ }^{91}$ Furthermore, the depletion of USP18 leads to a strong increase in the levels and activity of miR-7, and this activity in turn decreases the expression of EGFR, leading to apoptosis and control of cancer cells. ${ }^{92}$

Hong et $a I^{93}$ found that IFN- $\gamma$ can induce USP18 in tumor cells and that this protein plays an important role in inhibiting tumorigenesis and maintaining antitumor immunity. Increasing the expression of USP18 in tumor cells suppresses tumorigenesis, whereas reducing its expression stimulates tumor development and decrease immunosurveillance. Moreover, USP18 expression in tumor cells regulates the exogenous production of IFN- $\gamma$ and the persistence of antigen-specific CTLs in the tumor microenvironment. ${ }^{93}$

A recent study showed that USP18 can predict the survival of patients with MIBC. Longer cancer-specific survival is associated with decreased expression of USP18 with or without the expression of the DGCR2 gene. ${ }^{94}$

\section{Conclusion}

In summary, USP18 is a novel protein that can affect cell viability at various cellular levels. Targeting this molecule may

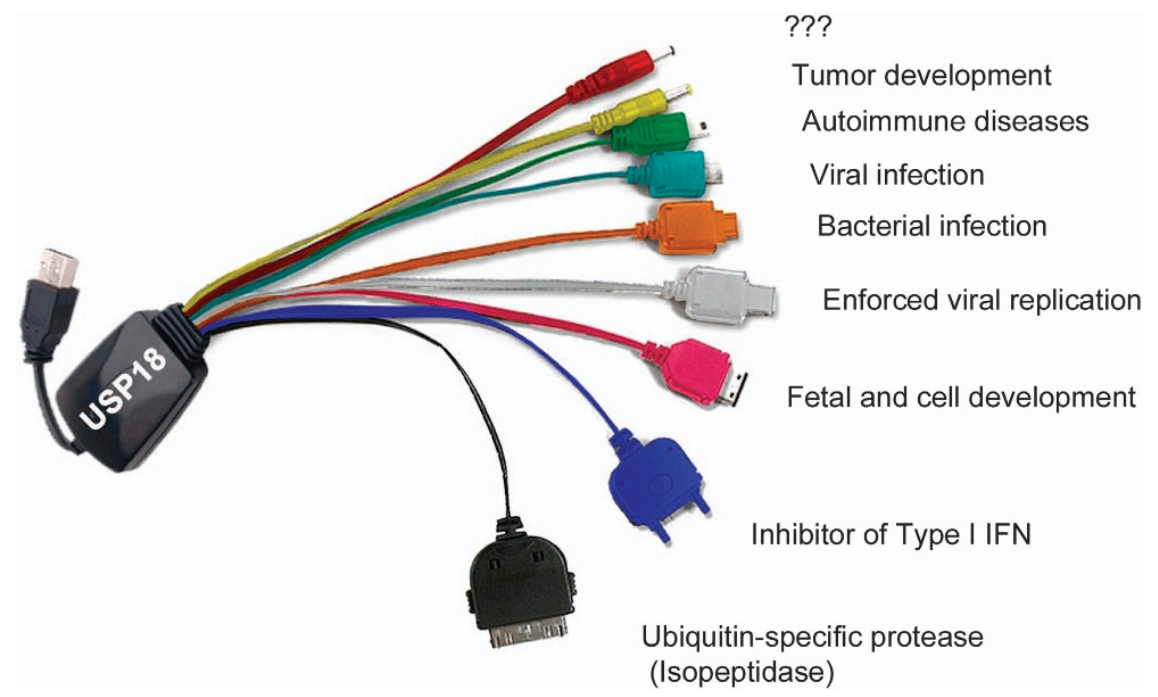

Figure 4 Multiple functions of USP18. The figure shows the multiple immunological and biological functions of USP18 in cell and organ development, infection, autoimmunity and tumor immunology 
help to improve the immune system response and avoid many (auto)-immune diseases, either by up- or downregulation of USP18 expression (Figure 4). For instance, some vaccines should be weakened and not completely inactivated in order to generate memory $B$ cells. According to this fact, upregulation of USP18 in macrophages and DCs will allow more sufficient amount of antigen and thereby may be useful during vaccine administration. Vice versa, downregulation of USP18 expression in antigen-presenting cells can be helpful not only to increase the antiviral signaling of IFN-I but also to reduce the amount of autoantigen and induction of autoimmune diseases. In both cases, we should take into consideration the yin and yang effect of IFN-I. ${ }^{95}$

For tumor treatment, the role of USP18 is still controversial. On the one hand, USP18 has a negative role either by inhibiting the antitumor effect of IFN-I or by accelerating the cell proliferation. On the other hand, it regulates the exogenous production of IFN- $\gamma$ and the persistence of antigen-specific CTLs in the tumor microenvironment, which suppresses tumorigenesis. According to that, further studies are still needed in order to show whether up- or downregulation of USP18 in each specific tumor type is beneficial or not.

In general, owing to the multiple functions of USP18 and its expression in various cell types, it would be complicated to generate one drug that can be used to treat all the diseases in which USP18 is involved. We believe that this difficulty can be resolved by using targeted drug delivery. Such technology would be the best strategy to overcome the protein's wide expression and will allow recovery from various types of immunological dysfunctions.

\section{Conflict of Interest}

The authors declare no conflict of interest.

Acknowledgements. We thank Mouhammad Wasseem Shaabani for his contribution in designing figures.

1. Francois-Newton V, Magno de Freitas Almeida G, Payelle-Brogard B, Monneron D, Pichard-Garcia L, Piehler J et al. USP18-based negative feedback control is induced by type I and type III interferons and specifically inactivates interferon alpha response. PLOS One 2011; 6: e22200.

2. Burkart C, Arimoto K, Tang T, Cong X, Xiao N, Liu YC et al. Usp18 deficient mammary epithelial cells create an antitumour environment driven by hypersensitivity to IFN-lambda and elevated secretion of Cxcl10. EMBO Mol Med 2013; 5: 967-982.

3. Liu LQ, Ilaria R Jr., Kingsley PD, Iwama A, van Etten RA, Palis J et al. A novel ubiquitinspecific protease, UBP43, cloned from leukemia fusion protein AML1-ETO-expressing mice, functions in hematopoietic cell differentiation. Mol Cell Biol 1999; 19: 3029-3038.

4. Schwer H, Liu LQ, Zhou L, Little MT, Pan Z, Hetherington CJ et al. Cloning and characterization of a novel human ubiquitin-specific protease, a homologue of murine UBP43 (Usp18). Genomics 2000; 65: 44-52.

5. Zhang X, Shin J, Molitor TW, Schook LB, Rutherford MS. Molecular responses of macrophages to porcine reproductive and respiratory syndrome virus infection. Virology 1999; 262: 152-162.

6. Kang $\mathrm{D}$, Jiang $\mathrm{H}, \mathrm{Wu} \mathrm{Q}$, Pestka S, Fisher PB. Cloning and characterization of human ubiquitin-processing protease-43 from terminally differentiated human melanoma cells using a rapid subtraction hybridization protocol RaSH. Gene 2001; 267: 233-242.

7. Baker RT, Wang XW, Woollatt E, White JA, Sutherland GR. Identification, functional characterization, and chromosomal localization of USP15, a novel human ubiquitin-specific protease related to the UNP oncoprotein, and a systematic nomenclature for human ubiquitin-specific proteases. Genomics 1999; 59: 264-274.

8. Ye Y, Scheel H, Hofmann K, Komander D. Dissection of USP catalytic domains reveals five common insertion points. Mol Biosyst 2009; 5: 1797-1808.
9. Malakhova OA, Kim KI, Luo JK, Zou W, Kumar KG, Fuchs SY et al. UBP43 is a novel regulator of interferon signaling independent of its ISG15 isopeptidase activity. EMBO J 2006; 25: 2358-2367.

10. Dauphinee SM, Richer E, Eva MM, McIntosh F, Paquet M, Dangoor D et al. Contribution of increased ISG15, ISGylation and deregulated type I IFN signaling in Usp18 mutant mice during the course of bacterial infections. Genes Immun 2014; 15: 282-292.

11. Richer E, Prendergast C, Zhang DE, Qureshi ST, Vidal SM, Malo D. N-ethyl-N-nitrosoureainduced mutation in ubiquitin-specific peptidase 18 causes hyperactivation of IFN-alphass signaling and suppresses STAT4-induced IFN-gamma production, resulting in increased susceptibility to Salmonella typhimurium. J Immunol 2010; 185: 3593-3601.

12. Burkart $\mathrm{C}$, Fan JB, Zhang DE. Two independent mechanisms promote expression of an $\mathrm{N}$-terminal truncated USP18 isoform with higher DelSGylation activity in the nucleus. J Biol Chem 2012; 287: 4883-4893.

13. Malakhov MP, Malakhova OA, Kim KI, Ritchie KJ, Zhang DE. UBP43 (USP18) specifically removes ISG15 from conjugated proteins. J Biol Chem 2002; 277: 9976-9981.

14. Honke N, Shaabani N, Cadeddu G, Sorg UR, Zhang DE, Trilling M et al. Enforced viral replication activates adaptive immunity and is essential for the control of a cytopathic virus. Nat Immunol 2011; 13: 51-57.

15. Honke N, Shaabani N, Zhang DE, lliakis G, Xu HC, Haussinger D et al. Usp18 driven enforced viral replication in dendritic cells contributes to break of immunological tolerance in autoimmune diabetes. PLoS Pathog 2013; 9: e1003650.

16. Liu X, Li H, Zhong B, Blonska M, Gorjestani S, Yan M et al. USP18 inhibits NF-kappaB and NFAT activation during Th17 differentiation by deubiquitinating the TAK1-TAB1 complex. $J$ Exp Med 2013; 210: 1575-1590.

17. Randall G, Chen L, Panis M, Fischer AK, Lindenbach BD, Sun J et al. Silencing of USP18 potentiates the antiviral activity of interferon against hepatitis $C$ virus infection. Gastroenterology 2006; 131: 1584-1591.

18. Ritchie KJ, Malakhov MP, Hetherington CJ, Zhou L, Little MT, Malakhova OA et al. Dysregulation of protein modification by ISG15 results in brain cell injury. Genes Dev 2002; 16: 2207-2212

19. Li L, Lei QS, Zhang SJ, Kong LN, Qin B. Suppression of USP18 potentiates the anti-HBV activity of interferon alpha in HepG2.2.15 cells via JAK/STAT signaling. PLoS One 2016; 11: e0156496.

20. Li XL, Blackford JA, Judge CS, Liu M, Xiao W, Kalvakolanu DV et al. RNase-L-dependent destabilization of interferon-induced mRNAs. A role for the 2-5 A system in attenuation of the interferon response. J Biol Chem 2000; 275: 8880-8888.

21. Kim KI, Malakhova OA, Hoebe K, Yan M, Beutler B, Zhang DE. Enhanced antibacterial potential in UBP43-deficient mice against Salmonella typhimurium infection by up-regulating type I IFN signaling. J Immunol 2005; 175: 847-854.

22. MacParland SA, Ma XZ, Chen L, Khattar R, Cherepanov V, Selzner M et al. Lipopolysaccharide and tumor necrosis factor Alpha inhibit interferon signaling in hepatocytes by increasing ubiquitin-like protease 18 (USP18) expression. J Virol 2016; 90: $5549-5560$.

23. Yang Z, Xian H, Hu J, Tian S, Qin Y, Wang RF et al. USP18 negatively regulates NF-kappaB signaling by targeting TAK1 and NEMO for deubiquitination through distinct mechanisms. SCi Rep 2015; 5: 12738.

24. Tokarz S, Berset C, La Rue J, Friedman K, Nakayama K, Zhang DE et al. The ISG15 isopeptidase UBP43 is regulated by proteolysis via the SCFSkp2 ubiquitin ligase. J Biol Chem 2004; 279: 46424-46430.

25. Frescas D, Pagano M. Deregulated proteolysis by the F-box proteins SKP2 and beta-TrCP: tipping the scales of cancer. Nat Rev Cancer 2008; 8: 438-449.

26. Malakhova OA, Yan M, Malakhov MP, Yuan Y, Ritchie KJ, Kim KI et al. Protein ISGylation modulates the JAK-STAT signaling pathway. Genes Dev 2003; 17: 455-460.

27. Potu $\mathrm{H}$, Sgorbissa A, Brancolini C. Identification of USP18 as an important regulator of the susceptibility to IFN-alpha and drug-induced apoptosis. Cancer Res 2010; 70: 655-665.

28. Yim HY, Yang Y, Lim JS, Lee MS, Zhang DE, Kim KI. The mitochondrial pathway and reactive oxygen species are critical contributors to interferon-alpha/beta-mediated apoptosis in Ubp43-deficient hematopoietic cells. Biochem Biophys Res Commun 2012; 423: $436-440$.

29. Rempel LA, Austin KJ, Ritchie KJ, Yan M, Shen M, Zhang DE et al. Ubp43 gene expression is required for normal Isg15 expression and fetal development. Reprod Biol Endocrinol 2007; 5: 13.

30. Knobeloch KP, Utermohlen O, Kisser A, Prinz M, Horak I. Reexamination of the role of ubiquitin-like modifier ISG15 in the phenotype of UBP43-deficient mice. Mol Cell Biol 2005; 25: 11030-11034

31. Yim HY, Park C, Lee YD, Arimoto K, Jeon R, Baek SH et al. Elevated response to type I IFN enhances RANKL-mediated osteoclastogenesis in Usp18-knockout mice. J Immunol 2016; 196: 3887-3895.

32. Ritchie KJ, Hahn CS, Kim KI, Yan M, Rosario D, Li L et al. Role of ISG15 protease UBP43 (USP18) in innate immunity to viral infection. Nat Med 2004; 10: 1374-1378.

33. Ketscher L, Hannss R, Morales DJ, Basters A, Guerra S, Goldmann T et al. Selective inactivation of USP18 isopeptidase activity in vivo enhances ISG15 conjugation and viral resistance. Proc Natl Acad Sci USA 2015; 112: 1577-1582.

34. Cong XL, Lo MC, Reuter BA, Yan M, Fan JB, Zhang DE. Usp18 promotes conventional CD11b+ dendritic cell development. J Immunol 2012; 188: 4776-4781.

35. Dong C. TH17 cells in development: an updated view of their molecular identity and genetic programming. Nat Rev Immunol 2008; 8: 337-348. 
36. King RW, Deshaies RJ, Peters JM, Kirschner MW. How proteolysis drives the cell cycle. Science 1996; 274: 1652-1659.

37. Zhu Y, Carroll M, Papa FR, Hochstrasser M, D'Andrea AD. DUB-1, a deubiquitinating enzyme with growth-suppressing activity. Proc Natl Acad Sci USA 1996; 93: 3275-3279.

38. Finley D, Chau V. Ubiquitination. Annu Rev Cell Biol 1991; 7: 25-69.

39. Miranda M, Dionne KR, Sorkina T, Sorkin A. Three ubiquitin conjugation sites in the amino terminus of the dopamine transporter mediate protein kinase $\mathrm{C}$-dependent endocytosis of the transporter. Mol Biol Cell 2007; 18: 313-323.

40. Verma IM, Stevenson JK, Schwarz EM, Van Antwerp D, Miyamoto S. Rel/NF-kappa B/I kappa B family: intimate tales of association and dissociation. Genes Dev 1995; 9: 2723-2735.

41. Rock KL, Gramm C, Rothstein L, Clark K, Stein R, Dick L et al. Inhibitors of the proteasome block the degradation of most cell proteins and the generation of peptides presented on $\mathrm{MHC}$ class I molecules. Cell 1994; 78: 761-771.

42. Hochstrasser M. Ubiquitin-dependent protein degradation. Annu Rev Genet 1996; 30 405-439.

43. Wilkinson KD. Regulation of ubiquitin-dependent processes by deubiquitinating enzymes. FASEB J 1997; 11: 1245-1256.

44. Narasimhan J, Potter JL, Haas AL. Conjugation of the 15-kDa interferon-induced ubiquitin homolog is distinct from that of ubiquitin. J Biol Chem 1996; 271: 324-330.

45. Ritchie KJ, Zhang DE. ISG15: the immunological kin of ubiquitin. Semin Cell Dev Biol 2004; 15: $237-246$

46. Yuan W, Krug RM. Influenza B virus NS1 protein inhibits conjugation of the interferon (IFN)induced ubiquitin-like ISG15 protein. EMBO J 2001; 20: 362-371.

47. Kim KI, Giannakopoulos NV, Virgin HW, Zhang DE. Interferon-inducible ubiquitin E2, Ubc8, is a conjugating enzyme for protein ISGylation. Mol Cell Biol 2004; 24: 9592-9600.

48. Zhao C, Beaudenon SL, Kelley ML, Waddell MB, Yuan W, Schulman BA et al. The UbcH8 ubiquitin E2 enzyme is also the E2 enzyme for ISG15, an IFN-alpha/beta-induced ubiquitinlike protein. Proc Natl Acad Sci USA 2004; 101: 7578-7582.

49. Dao CT, Zhang DE. ISG15: a ubiquitin-like enigma. Front Biosci 2005; 10: 2701-2722.

50. Zou W, Zhang DE. The interferon-inducible ubiquitin-protein isopeptide ligase (E3) EFP also functions as an ISG15 E3 ligase. J Biol Chem 2006; 281: 3989-3994.

51. Ait-Ali T, Wilson AW, Finlayson H, Carre W, Ramaiahgari SC, Westcott DG et al. Functional analysis of the porcine USP18 and its role during porcine arterivirus replication. Gene 2009; 439: $35-42$.

52. Zhang X, Bogunovic D, Payelle-Brogard B, Francois-Newton V, Speer SD, Yuan C et al. Human intracellular ISG15 prevents interferon-alpha/beta over-amplification and auto-inflammation. Nature 2015; 517 : 89-93.

53. Speer SD, Li Z, Buta S, Payelle-Brogard B, Qian L, Vigant F et al. ISG15 deficiency and increased viral resistance in humans but not mice. Nat Commun 2015; 7: 11496.

54. Warsi J, Hosseinzadeh Z, Elvira B, PelzI L, Shumilina E, Zhang DE et al. USP18 Sensitivity of Peptide Transporters PEPT1 and PEPT2. PLoS One 2015; 10: e0129365.

55. Ivashkiv LB, Donlin LT. Regulation of type I interferon responses. Nat Rev Immunol 2014; 14 : 36-49.

56. Osiak A, Utermohlen O, Niendorf S, Horak I, Knobeloch KP. ISG15, an interferon-stimulated ubiquitin-like protein, is not essential for STAT1 signaling and responses against vesicular stomatitis and lymphocytic choriomeningitis virus. Mol Cell Biol 2005; 25: 6338-6345.

57. Kim KI, Yan M, Malakhova O, Luo JK, Shen MF, Zou W et al. Ube1L and protein ISGylation are not essential for alpha/beta interferon signaling. Mol Cell Biol 2006; 26: 472-479.

58. Francois-Newton V, Livingstone M, Payelle-Brogard B, Uze G, Pellegrini S. USP18 establishes the transcriptional and anti-proliferative interferon alpha/beta differential. Biochem J 2012; 446: 509-516.

59. Zou W, Kim JH, Handidu A, Li X, Kim KI, Yan M et al. Microarray analysis reveals that Type I interferon strongly increases the expression of immune-response related genes in Ubp43 (Usp18) deficient macrophages. Biochem Biophys Res Commun 2007; 356: 193-199.

60. Xu D, Lillico SG, Barnett MW, Whitelaw CB, Archibald AL, Ait-Ali T. USP18 restricts PRRSV growth through alteration of nuclear translocation of NF-kappaB p65 and p50 in MARC-145 cells. Virus Res 2012; 169: 264-267.

61. Kim JH, Luo JK, Zhang DE. The level of hepatitis B virus replication is not affected by protein ISG15 modification but is reduced by inhibition of UBP43 (USP18) expression. J Immunol 2008; 181: 6467-6472

62. Zhu Y, Qin B, Xiao C, Lu X, Chen L. Cell-type specific interferon stimulated gene staining in liver underlies response to interferon therapy in chronic HBV infected patients. Dig Dis Sci 2012; 57: 2355-2361.

63. Lenschow DJ, Giannakopoulos NV, Gunn LJ, Johnston C, O'Guin AK, Schmidt RE et al. Identification of interferon-stimulated gene 15 as an antiviral molecule during Sindbis virus infection in vivo. J Virol 2005; 79: 13974-13983.

64. Okumura A, Lu G, Pitha-Rowe I, Pitha PM. Innate antiviral response targets HIV-1 release by the induction of ubiquitin-like protein ISG15. Proc Natl Acad Sci USA 2006; 103: 1440-1445.

65. Lenschow DJ, Lai C, Frias-Staheli N, Giannakopoulos NV, Lutz A, Wolff T et al. IFN-stimulated gene 15 functions as a critical antiviral molecule against influenza, herpes, and Sindbis viruses. Proc Natl Acad Sci USA 2007; 104: 1371-1376.

66. Ma XZ, Bartczak A, Zhang J, He W, Shalev I, Smil D et al. Protein interferon-stimulated gene 15 conjugation delays but does not overcome coronavirus proliferation in a model of fulminant hepatitis. J Virol 2014; 88: 6195-6204.
67. Asahina Y, Izumi N, Hirayama I, Tanaka T, Sato M, Yasui $Y$ et al. Potential relevance of cytoplasmic viral sensors and related regulators involving innate immunity in antiviral response. Gastroenterology 2008; 134: 1396-1405.

68. Chen L, Sun J, Meng L, Heathcote J, Edwards AM, McGilvray ID. ISG15, a ubiquitin-like interferon-stimulated gene, promotes hepatitis $C$ virus production in vitro: implications for chronic infection and response to treatment. J Gen Virol 2010; 91: 382-388.

69. Li Y, Li S, Duan X, Liu B, Yang C, Zeng P et al. Activation of endogenous type I IFN signaling contributes to persistent HCV infection. Rev Med Virol 2014; 24: 332-342.

70. Rubartelli A, Lotze MT, Latz E, Manfredi A. Mechanisms of sterile inflammation Front Immunol 2013; 4: 398.

71. Henrickson SE, Mempel TR, Mazo IB, Liu B, Artyomov MN, Zheng $\mathrm{H}$ et al. T cell sensing of antigen dose governs interactive behavior with dendritic cells and sets a threshold for $\mathrm{T}$ cell activation. Nat Immunol 2008; 9: 282-291.

72. Holler PD, Kranz DM. Quantitative analysis of the contribution of TCR/pepMHC affinity and CD8 to T cell activation. Immunity 2003; 18: 255-264.

73. Colonne PM, Sahni A, Sahni SK. Suppressor of cytokine signalling protein SOCS1 and UBP43 regulate the expression of type I interferon-stimulated genes in human microvascular endothelial cells infected with Rickettsia conorii. J Med Microbiol 2013; 62: 968-979.

74. Colonne PM, Sahni A, Sahni SK. Rickettsia conorii infection stimulates the expression of ISG15 and ISG15 protease UBP43 in human microvascular endothelial cells. Biochem Biophys Res Commun 2011; 416: 153-158.

75. Santin I, Eizirik DL. Candidate genes for type 1 diabetes modulate pancreatic islet inflammation and beta-cell apoptosis. Diabetes Obes Metab 2013; 15: 71-81.

76. Santin I, Moore F, Grieco FA, Marchetti P, Brancolini C, Eizirik DL. USP18 is a key regulator of the interferon-driven gene network modulating pancreatic beta cell inflammation and apoptosis. Cell Death Dis 2012; 3: e419.

77. Bendtzen K. Critical review: assessment of interferon-beta immunogenicity in multiple sclerosis. J Interferon Cytokine Res 2010; 30: 759-766.

78. Jacobs LD, Beck RW, Simon JH, Kinkel RP, Brownscheidle CM, Murray TJ et al. Intramuscular interferon beta-1a therapy initiated during a first demyelinating event in multiple sclerosis. CHAMPS Study Group. N Engl J Med 2000; 343: 898-904.

79. Goldmann T, Zeller N, Raasch J, Kierdorf K, Frenzel K, Ketscher L et al. USP18 lack in microglia causes destructive interferonopathy of the mouse brain. EMBO J 2015; 34 1612-1629.

80. Takata K, Ginhoux F. Poised for action: USP18 restrains microglial activation in the white matter. EMBO J 2015; 34: 1603-1605.

81. Malhotra S, Bustamante MF, Perez-Miralles F, Rio J, Ruiz de Villa MC, Vegas E et al. Search for specific biomarkers of IFNbeta bioactivity in patients with multiple sclerosis. PLoS One 2011; 6: e23634.

82. Malhotra S, Morcillo-Suarez C, Nurtdinov R, Rio J, Sarro E, Moreno M et al. Roles of the ubiquitin peptidase USP18 in multiple sclerosis and the response to interferon-beta treatment. Eur J Neurol 2013; 20: 1390-1397.

83. Yan M, Luo JK, Ritchie KJ, Sakai I, Takeuchi K, Ren R et al. Ubp43 regulates BCR-ABL leukemogenesis via the type 1 interferon receptor signaling. Blood 2007; 110: 305-312.

84. Sgorbissa A, Tomasella A, Potu H, Manini I, Brancolini C, Type I. IFNs signaling and apoptosis resistance in glioblastoma cells. Apoptosis 2011; 16: 1229-1244.

85. Guo Y, Dolinko AV, Chinyengetere F, Stanton B, Bomberger JM, Demidenko $E$ et al. Blockade of the ubiquitin protease UBP43 destabilizes transcription factor PML/RARalpha and inhibits the growth of acute promyelocytic leukemia. Cancer Res 2010; 70: 9875-9885.

86. Guo Y, Chinyengetere F, Dolinko AV, Lopez-Aguiar A, Lu Y, Galimberti F et al. Evidence for the ubiquitin protease UBP43 as an antineoplastic target. Mol Cancer Ther 2012; 11: 1968-1977.

87. Shahidul Makki M, Cristy Ruteshouser E, Huff V. Ubiquitin specific protease 18 (Usp18) is a WT1 transcriptional target. Exp Cell Res 2013; 319: 612-622.

88. Feng Q, Sekula D, Guo Y, Liu X, Black CC, Galimberti F et al. UBE1L causes lung cancer growth suppression by targeting cyclin D1. Mol Cancer Ther 2008; 7: 3780-3788.

89. Hoeller D, Hecker CM, Dikic I. Ubiquitin and ubiquitin-like proteins in cancer pathogenesis. Nat Rev Cancer 2006; 6: 776-788.

90. Pitha-Rowe I, Petty WJ, Feng Q, Koza-Taylor PH, Dimattia DA, Pinder L et al. Microarray analyses uncover UBE1L as a candidate target gene for lung cancer chemoprevention. Cancer Res 2004; 64: 8109-8115.

91. Manini I, Sgorbissa A, Potu H, Tomasella A, Brancolini C. The DelSGylase USP18 limits TRAIL-induced apoptosis through the regulation of TRAIL levels: Cellular levels of TRAIL influences responsiveness to TRAlL-induced apoptosis. Cancer Biol Ther 2013; 14 $1158-1166$.

92. Duex JE, Comeau L, Sorkin A, Purow B, Kefas B. Usp18 regulates epidermal growth factor (EGF) receptor expression and cancer cell survival via microRNA-7. J Biol Chem 2011; 286: 25377-25386.

93. Hong B, Li H, Lu Y, Zhang M, Zheng Y, Qian J et al. USP18 is crucial for IFN-gammamediated inhibition of B16 melanoma tumorigenesis and antitumor immunity. Mol Cancer 2014; 13: 132.

94. Kim YH, Kim WT, Jeong P, Ha YS, Kang HW, Yun SJ et al. Novel combination markers for predicting survival in patients with muscle invasive bladder cancer: USP18 and DGCR2. J Korean Med Sci 2014; 29: 351-356.

95. Trinchieri G. Type I interferon: friend or foe? J Exp Med 2010; 207: 2053-2063. 